\title{
Elektronenoptischer Phasenkontrast
}

\section{Ansatz für eine quantitative Theorie}

\section{REIMER}

Physikalisches Institut der Universität Münster/Westf.

(Z. Naturforschg. 21 a, 1489-1499 [1966] ; eingegangen am 16. Mai 1966)

\begin{abstract}
Mit der Bonsschen Näherung als Ausgangspunkt wird die Wirkung des Öffnungsfehlers und einer Defokussierung auf den Phasenkontrast eines phasenschiebenden Scheibchens von 5 bis $40 \AA$ Durchmesser berechnet und die Frage diskutiert, inwieweit derartige Objekte durch Phasenkontrast abgebildet werden können. Es wird ferner der Versuch unternommen, den Phasenkontrast auch quantitativ auf der Basis der Elektronenstreutheorie zu erfassen. Bei derartig kleinen Objekten muß man auch den Aufbau aus Einzelatomen berücksichtigen. Die Diskussion zeigt, da $\beta$ man jedoch bei den kleinen Aperturwinkeln, die zur Abbildung benutzt werden, keinen nennenswerten Einfluß des atomaren Aufbaues findet. Die Annahme einer phasenschiebenden Scheibe mit einem inneren Potential stellt bei Scheiben mit 10 bis $20 \AA$ Durchmesser aus einer Monolage von Atomen noch eine befriedigende Näherung dar.
\end{abstract}

Durch die Arbeiten von Albert u. a. ${ }^{1}$ und Thon ${ }^{2,3}$ sowie die Bemühungen zur Verbesserung des Auflösungsvermögens der Elektronenmikroskope - sei es mit Hilfe von Kondensor-Objektiv-Einfeldlinsen ${ }^{4,5}$ oder Hoppe-Lenz-Platten ${ }^{6-8}$ - ist die Ausnutzung des Phasenkontrastes in der Elektronenmikroskopie in den Brennpunkt des Interesses gerückt. Wieweit die letztgenannten Möglichkeiten sich praktisch realisieren lassen, muß die Entwicklung in den nächsten Jahren zeigen. Der Phasenkontrast durch Wirkung des Öffnungsfehlers und einer Defokussierung ${ }^{9,1}$ spielt bei der Kontrastentstehung in den zur Zeit zur Verfügung stehenden Hochleistungs-Elektronenmikroskopen jedoch schon eine entscheidende Rolle. Man wird in der nächsten Zeit auch mit quantitativen Messungen und Bildinterpretationen rechnen können. Deshalb soll in der vorliegenden Arbeit der Versuch unternommen werden, einen Beitrag zur quantitativen Erfassung des Phasenkontrastes zu liefern und ihn am speziellen Beispiel eines phasenschiebenden Scheibchens zu erläutern. Ferner wird über Rechnungen berichtet, welche die Zusammensetzung kleiner Phasenobjekte aus Einzelatomen berücksichtigen.

1 L. Albert, R. Schneider u. H. Fischer, Z. Naturforschg. 19 a, 1120 [1964].

2 F. THON, Z. Naturforschg. 20 a, 154 [1965].

3 F. Thon, Proc. 3rd European Regional Conf. Electron Microscopy, Prag, Vol. A, 127 [1964].

${ }^{4}$ E. Ruska, Naturw. Rundschau 17, 125 [1964] ; Optik 22, 319 [1965].

5 W. D. Riecke, V. Intern. Congr. Electron Microscopy Philadelphia, Vol. I, KK-5 [1962].
Der elektronenoptische Brechungsindex berechnet sich aus dem „inneren Potential“ $U_{\mathrm{i}}$ nach der Formel ( $U_{0}=$ Beschleunigungsspannung der Elektronen $)$

$$
\begin{aligned}
n=\frac{\lambda_{0}}{\lambda_{\mathrm{i}}} & =\left\{\frac{\left(U_{0}+U_{\mathrm{i}}\right)\left(1+\frac{e\left(U_{0}+U_{\mathrm{i}}\right)}{m c^{2}}\right)}{U_{0}\left(1+\frac{e U_{0}}{m c^{2}}\right)}\right\}^{1 / 2} \\
& \cong \sqrt{1+\frac{U_{\mathrm{i}}}{U_{0}} \cong 1+\frac{1}{2} \frac{U_{\mathrm{i}}}{U_{0}} .}
\end{aligned}
$$

Der in einer Schicht der Dicke $D$ hervorgerufene Gangunterschied beträgt

$$
\delta=\frac{2 \pi}{\lambda}(n-1) D[\mathrm{rad}] .
$$

Die Phasendrehung $\delta$ kann man erreichen, wenn entweder bei konstanter Schichtdicke $D$ das innere Potential durch Dichteschwankungen kleiner oder größer als die Umgebung ist, oder wenn sich bei konstantem $U_{\mathrm{i}}$ die Schichtdicke lokal ändert (Abb. 1). Aus (1) und (2) folgt daher

$$
\delta=\pi \Delta\left(U_{\mathrm{i}} D\right) /\left(\lambda U_{0}\right) .
$$

Das innere Potential $U_{\mathrm{i}}$ liegt in der Größenordnung $10-20$ Volt. Bei $U_{\mathrm{i}}=10 \mathrm{~V}, \lambda=0,037 \AA$

6 W. Hoppe, Naturwiss. 48, 736 [1961] ; Optik 20, 599 [1963].

7 F. Lenz, Z. Phys. 172, 498 [1963] ; Optik 21, 489 [1964].

8 K. H. von Grote, G. Möllenstedt u. R. Speidel, Optik 22, 252 [1965].

9 O. Scherzer, J. Appl. Phys. 20, 20 [1949]. 
(100 kV-Elektronen) beträgt $(n-1)=5 \cdot 10^{-5}$ und eine Phasenverschiebung von $\pi / 2\left(=90^{\circ}\right)$ wird bereits durch eine Schicht von $185 \AA$ erzeugt.

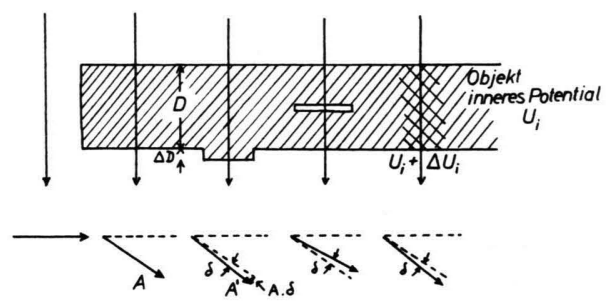

Abb. 1. Verschiedene Möglichkeiten, in denen sich ein „Phasenobjekt" von der Umgebung durch Differenzen $\delta$ in der Phasenschiebung unterscheidet.

Die Wellenlänge $\lambda_{\mathrm{i}}$ im Objekt ist nach (1) kleiner als im Vakuum. Daher läuft die Phase der Welle mit der Amplitude $A$ im Objekt derjenigen im Vakuum nach. In einer Säule des Objektes in Elektronenstrahlrichtung ( $z$-Richtung) kann man den Verlauf der Phase in einem Amplituden-Phasen-Diagramm darstellen (Abb. 1). Hinter dem Phasenobjekt ist die Amplitude um den Winkel $\delta$ gedreht. Der $\mathrm{Zu}$ satzbeitrag $A \cdot \delta$ gegenüber der Umgebung ist um $90^{\circ}$ gegenüber der Amplitude des ungestörten Gebietes verschoben. In der bildseitigen Brennebene des Objektivs entsteht bei jeder Abbildung ein 1. Beugungsbild. Die durch das Objekt gehende Strahlung (parallel einfallendes Bündel vorausgesetzt) wird praktisch im Brennpunkt vereinigt. Eine sehr geringe Verbreiterung erfolgt nur durch Beugung infolge der Bündelbegrenzung durch die Objektblende oder den bestrahlten Bereich. Praktisch liegen jedoch in der Elektronenmikroskopie Beleuchtungsaperturen der Größenordnung $10^{-3}$ bis $10^{-5}$ je nach Erregung des Kondensors und Größe der Kondensorblende vor. Dies führt zu einer entsprechenden Verbreiterung des Brennfleckes.

Die um $90^{\circ}$ phasenverschobene Komponente $A \cdot \delta$ des Phasenobjektes wird aber mit abnehmendem Durchmesser $2 r$ der Phasenstruktur zu einer breiter werdenden Beugungsfigur führen (Abb. 3). Bei der Superposition im Bildpunkt ist wieder die Amplitude des Phasenobjektes um $\delta$ gegenüber der Umgebung verschoben. Der Betrag der Amplitude bleibt unverändert $\left(|A|=\left|A^{\prime}\right|\right)$. Die Phasenstruktur ist unsichtbar.

Elektronen, welche unter einem Winkel $\vartheta$ zum Primärstrahl gestreut werden und unter diesem Winkel die Objektivlinse durchlaufen, erleiden eine Phasenverschiebung ${ }^{9} \Delta$ durch den Öffnungsfehler
( $C_{\ddot{0}}=$ Öffnungsfehlerkonstante $\cong 4,5 \mathrm{~mm}$ beim Elmiskop I) und durch die Defokussierung $\Delta f$

$$
\Delta=\frac{\pi}{2 \lambda}\left(C_{\ddot{0}} \vartheta^{4}-2 \Delta f \vartheta^{2}\right)
$$

Diese Phasenverschiebung durch Öffnungsfehler und Defokussierung dreht die um $90^{\circ}$ verschobene Komponente $A \cdot \delta$, wodurch bei einer Überlagerung im Bildpunkt $|A| \neq\left|A^{\prime}\right|$ wird, und die Phasenstruktur wird je nach dem Vorzeichen von $\delta$ und der Phasenschiebung $\Delta$ heller oder dunkler als die Umgebung abgebildet.

Für eine quantitative Theorie des Phasenkontrastes ist es zunächst erforderlich, die Beugungsamplitude $F(\vartheta)$ der Phasenstruktur als Funktion des Streuwinkels $\vartheta \mathrm{zu}$ ermitteln. Darauf kann die Phasenschiebung nach (4) angewandt werden, indem mit dem Phasenfaktor $e^{i \Delta}$ multipliziert wird. Anschließend erfolgt die Bildrekonstruktion durch Fourier-Transformation des so in der Phase modifizierten Beugungsbildes. Ein weiterer Eingriff erfolgt durch die Aperturbegrenzung mittels der Objektivaperturblende in der Ebene des Beugungsbildes.

\section{Phasenkontrast in Bornscher Näherung}

Es gibt jedoch keine reinen Phasenobjekte in der Elektronenmikroskopie, sondern die elastische und unelastische Streuung ruft eine Intensitätsschwächung im Endbild hervor, indem alle Elektronen, welche in Winkel größer als die Objektivapertur $\alpha$ gestreut werden, von der Aperturblende zurückgehalten werden. Da die elastische Elektronenstreuung mit Hilfe der quantenmechanischen Borsschen Näherung beschrieben werden kann, ist diese auch für eine Berechnung der Amplitudenverteilung des Beugungsbildes einer Phasenstruktur anzuwenden.

Die Lösung der SchröDinger-Gleichung

$$
\Delta \psi+\frac{2 \text { e } m}{\hbar^{2}}\left(U_{0}-U(\mathfrak{r})\right) \psi=0
$$

wird in Form einer einfallenden ebenen Welle $\psi^{0}$ und einer auslaufenden Kugelwelle angesetzt

$$
\psi=\psi^{0}+F(\vartheta)[\exp (2 \pi i f \mathfrak{r})] / r .
$$

Die Amplitude $F(\vartheta)$ der gestreuten Welle ergibt sich nach der 1. BorNschen Näherung:

$$
F(\vartheta)=-\frac{1}{4 \pi} \frac{2 e m}{\hbar^{2}} \int \exp \left(2 \pi i\left(\mathfrak{f}-\mathfrak{f}_{0}\right) \cdot \mathfrak{r}\right) \cdot U(\mathfrak{r}) \mathrm{d} \tau
$$


mit $\mathfrak{f}$ als Wellenzahlvektor der auslaufenden und $\mathfrak{f}_{0}$ der einfallenden Welle. Dabei ist

$$
\begin{gathered}
|\mathfrak{f}|=1 / \lambda ; \\
\left(\mathfrak{f}-\mathfrak{f}_{0}\right)=(1 / \lambda)\left(e-\mathfrak{e}_{0}\right) ; \\
\mathfrak{e} \text { und } \mathfrak{e}_{0} \text { Einheitsvektoren, } \\
\left|\mathfrak{f}-\mathfrak{f}_{0}\right|=(1 / \lambda) \sin \vartheta \cong \vartheta / \lambda .
\end{gathered}
$$

Die Integration erstreckt sich über den ganzen Streukörper mit dem Potential $U(\mathfrak{r})$. Setzt man hierfür ein abgeschirmtes Cou Lомв-Potential

$$
U(\mathrm{r})=U(r)=-(e Z / r) \exp (-r / R)
$$

mit $R$ als „Atomradius“ ein, so ist die Integration in (7) elementar ausführbar und liefert direkt den von LENZ ${ }^{10}$ angegebenen Ausdruck für die elastische Streuung eines Einzelatoms

$$
f(\vartheta)=\frac{2 Z R^{2}}{a_{\mathrm{H}}} \frac{1}{1+\left(\vartheta / \vartheta_{0}\right)^{2}}
$$

mit $a_{\mathrm{H}}=0,529 \AA$ als BoHRschen Wasserstoffradius und $\vartheta_{0}=\lambda /(2 \pi R)$ als „charakteristischen Winkel“. Da man die abgebeugte Intensität als Quadrat der Amplitude erhält, hat $f^{2}(\vartheta)=\mathrm{d} \sigma_{\mathrm{el}} / \mathrm{d} \Omega$ die Bedeutung eines differentiellen Streuquerschnittes ${ }^{10 a}$.

Geht man von der Streuung am Einzelatom zu einem zusammengesetzten Streukörper über (z. B. amorphe oder kristalline Schicht), so kann man berücksichtigen, daß $F(\vartheta)$ in (7) proportional zur Fourier-Transformierten des Potentials $U(\mathfrak{r})$ ist:

$$
F(\vartheta) \sim V_{\mathfrak{h}}=\int \exp (2 \pi i \mathfrak{h} \cdot \mathfrak{r}) \cdot U(\mathfrak{r}) \mathrm{d} \tau
$$

mit $\mathfrak{h}=\left(\mathfrak{f}-\mathfrak{f}_{0}\right)$ als Vektor des reziproken Raumes. Das Potential $U(\mathrm{r})$ läßt sich umgekehrt als FourierIntegral darstellen

$$
U(\mathfrak{r})=\int V_{\mathfrak{h}} \exp (-2 \pi i \mathfrak{h} \cdot \mathfrak{r}) \mathrm{d} \tau^{\prime}
$$

( $\mathrm{d} \tau^{\prime}$ Volumenelement des reziproken Raumes).

In kristallinen Objekten besteht $V_{\mathfrak{h}}$ und damit auch $F(\vartheta)$ aus einem diskreten Spektrum, da es nur an Gitterpunkten des reziproken Gitters von Null verschieden ist und man kann schreiben

$$
U(\mathfrak{r})=\sum_{\mathfrak{h}} V_{\mathfrak{h}} \exp (-2 \pi i \mathfrak{h} \cdot \mathfrak{r})
$$

summiert über alle Gitterpunkte $\mathfrak{h}$ des reziproken Gitters. Der Koeffizient $V_{0}=U_{\mathrm{i}}$ für $\mathfrak{h}=0$ dieser Entwicklung ist das „innere Potential“. Bei amorphen Objekten stellt $V(\mathfrak{h})$ die Fourier-Transformation der Atomdichteverteilung dar. Abb. 2 zeigt schema-

10 F. Lenz, Z. Naturforschg. 9 a, 185 [1954].

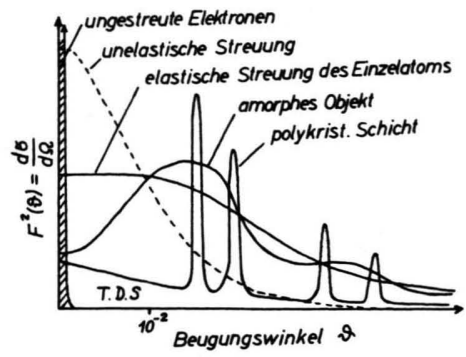

Abb. 2. Schematischer Verlauf der Streukurven $F^{2}(\vartheta)$ für amorphe und polykristalline Schichten, sowie für Einzelatome (Gas-Streuung) (T.D.S.=thermisch diffuse Streuung).

tisch, wie sich die elastische Streuung des Einzelatoms (10) bei kleinen Winkeln durch diese „Packungseffekte“ verändert. Weiter unten wird der Fall eines kristallinen und amorphen Phasenobjektes noch näher diskutiert. Zunächst sei der Spezialfall einer phasenschiebenden Scheibe mit dem Durchmesser $2 R$, der Dicke $D$ und dem inneren Potential $U_{\mathrm{i}}$ angenommen.

Da für die Streuung an der Phasenstruktur nur der Unterschied gegenüber der Umgebung zu berücksichtigen ist, hat man in (7) für $U(r)=U_{\mathrm{i}}$ innerhalb der Scheibe und $U(r)=0$ außerhalb der Scheibe zu setzen. Aus (7) ergibt sich dann für die gestreute Amplitude mit $A_{0}$ als Amplitude der einfallenden Welle (s. Abb. 3)

$$
\begin{aligned}
& A_{0} F(\vartheta)=\frac{A_{0}}{4 \pi} \frac{2 e m}{\hbar^{2}} \\
& \cdot \int_{0}^{R} \int_{-\pi}^{+\pi} \int_{0}^{D} U_{\mathrm{i}} \exp \left(\frac{2 \pi i}{\lambda} r \vartheta \cos \varphi\right) r \mathrm{~d} r \mathrm{~d} \varphi \mathrm{d} z .
\end{aligned}
$$

Mit der Relation

$$
\int_{-\pi}^{+\pi} \exp (i x \cos \varphi) \mathrm{d} \varphi=2 \pi J_{0}(x)
$$

( $J_{0}$ Bessel-Funktion 0. Ordnung) ergibt sich aus (14)

$$
A_{0} F(\vartheta)=\frac{A_{0} e m U_{\mathrm{i}} D}{\hbar^{2}} \int_{0}^{R} J_{0}\left(\frac{2 \pi}{\lambda} r \vartheta\right) r \mathrm{~d} r .
$$

Da $\int x J_{0}(x) \mathrm{d} x=x J_{1}(x)$ ist, folgt mit der Substitution $x=(2 \pi / \lambda) r \vartheta$

$$
A_{0} F(\vartheta)=\frac{A_{0} e m U_{\mathrm{i}} D \lambda R}{2 \pi \hbar^{2} \vartheta} J_{1}\left(\frac{2 \pi}{\lambda} R \vartheta\right) .
$$

Dieser Ausdruck gibt die um $90^{\circ}$ verschobene Amplitudenverteilung in der Beugungsebene wieder. Die

10a Mit $f(\vartheta)$ soll im folgenden die Streufunktion eines Einzelatoms und mit $F(\vartheta)$ diejenige eines zusammengesetzten Streukörpers unterschieden werden. 
unter dem Winkel $\vartheta$ gestreute Strahlung ist mit dem Phasenfaktor $e^{i \Delta}$ zu multiplizieren. Die Rücktransformation in die Bildebene (Abbildungsmaßstab 1:1) erhält man durch eine weitere Fourier-Transformation.

$$
B\left(r^{\prime}\right)=\frac{A_{0}}{\lambda} \int_{0}^{\alpha} \int_{-\pi}^{+\pi} F(\vartheta) \exp (i \Delta) \cdot \exp \left(-\frac{2 \pi i}{\lambda} r^{\prime} \vartheta \cos \chi\right) \vartheta \mathrm{d} \vartheta \mathrm{d} \chi .
$$

(Zur Bedeutung von $\chi$ s. Abb. 3, $\alpha=$ Objektivapertur). Der Faktor $1 / \lambda$ muß in (18) aus Normierungsgründen hinzugefügt werden. Nur dann bleibt die Elektronendichte, welche der Intensität und damit dem Amplitudenquadrat proportional ist, erhalten (s. a. Darstellung des entsprechenden optischen Problems ${ }^{11}$ ).

Einsetzen von (17) in (18) führt zu dem Ausdruck

$$
B\left(r^{\prime}\right)=A_{0} \frac{e m U_{\mathrm{i}} D R}{\hbar^{2}} \int_{0}^{\alpha} J_{1}\left(\frac{2 \pi}{\lambda} R \vartheta\right) J_{0}\left(\frac{2 \pi}{\lambda} r^{\prime} \vartheta\right) e^{i \Delta(\vartheta)} \mathrm{d} \vartheta=\left|B\left(r^{\prime}\right)\right| e^{i \Delta^{\prime}} .
$$

Nach Abb. 4 gilt $\left|B_{\text {ges }}\right|=A_{0}+\left|B\left(r^{\prime}\right)\right| \sin \Delta^{\prime}$, und da $e^{i \Delta}=\cos \Delta+i \sin \Delta$ ist, braucht in (19) nur der Term $\sin \Delta$ des Phasenfaktors berücksichtigt werden, um den Beitrag des Phasenkontrastes zu erhalten:

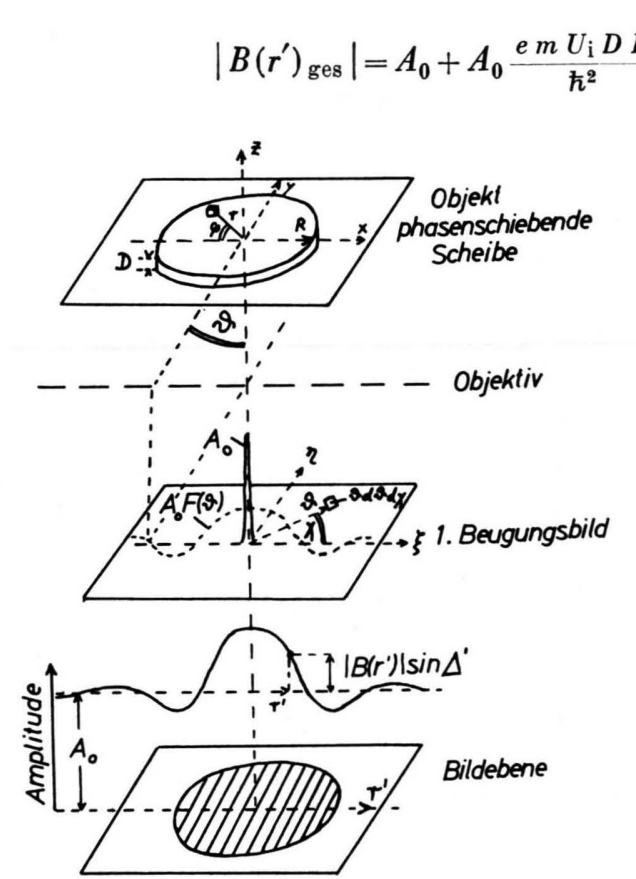

$\leftarrow$ Abb. 3. Zur Erläuterung der Koordinaten im Objekt, 1. Beugungsbild und der Bildebene.

Mit der Relation $U_{0} \lambda^{2}=h^{2} / 2$ e $m$ und

$$
\int_{0}^{\infty} J_{1}\left(\frac{2 \pi}{\lambda} R \vartheta\right) J_{0}\left(\frac{2 \pi}{\lambda} r^{\prime} \vartheta\right) \mathrm{d} \vartheta=\frac{\lambda}{2 \pi R}
$$

d. h. für den Fall ohne Aperturbegrenzung und ohne Phasenschiebung durch die Linse ergibt sich aus (20)

$$
B\left(r^{\prime}\right)_{\text {ges }}=A_{0}+i A_{0} \frac{\pi D U_{\mathrm{i}}}{\lambda U_{0}}=A_{0}+i A_{0} \cdot \delta
$$

mit der in (3) elementar abgeleiteten Phasenver-
Abb. 4. Uberlagerung der Amplitude $A_{0}$ und der vom Phasenobjekt gestreuten Amplitude $A_{0} \delta$ (um $90^{\circ}$ phasenverschoben), welche durch Einwirkung des Objektivs und der Abbildung in der Bildebene $\mathrm{zu} B\left(r^{\prime}\right) e^{i \Delta^{\prime}}$ modifiziert wurde. schiebung $\delta$ des Objektes. Damit ist nachgewiesen, wie auch theoretisch zu erwarten ist, daß mit der Borsschen Näherung (7) als Ausgangsgleichung und Berücksichtigung von nur einer FourIER-Komponenten $U_{\mathrm{i}}$ aus (12) bzw. (13) die klassische Gleichung (3) resultiert. Darüber hinaus liefert die Bonssche Näherung über die Gln. (7) bis (20) ein Verfahren durch Integration über eine beliebige Potentialverteilung $U(\mathfrak{r})$ den Phasenkontrast quantitativ zu berechnen.

11 M. Franzen, Interférences, diffraction et polarisation, in Handbuch der Physik, Bd. 24 (Hrsg. S. FLüGGE), Springer-Verlag, Berlin 1956. 


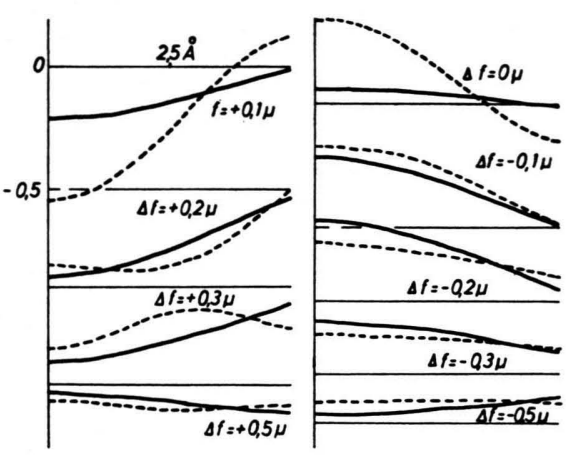

a)

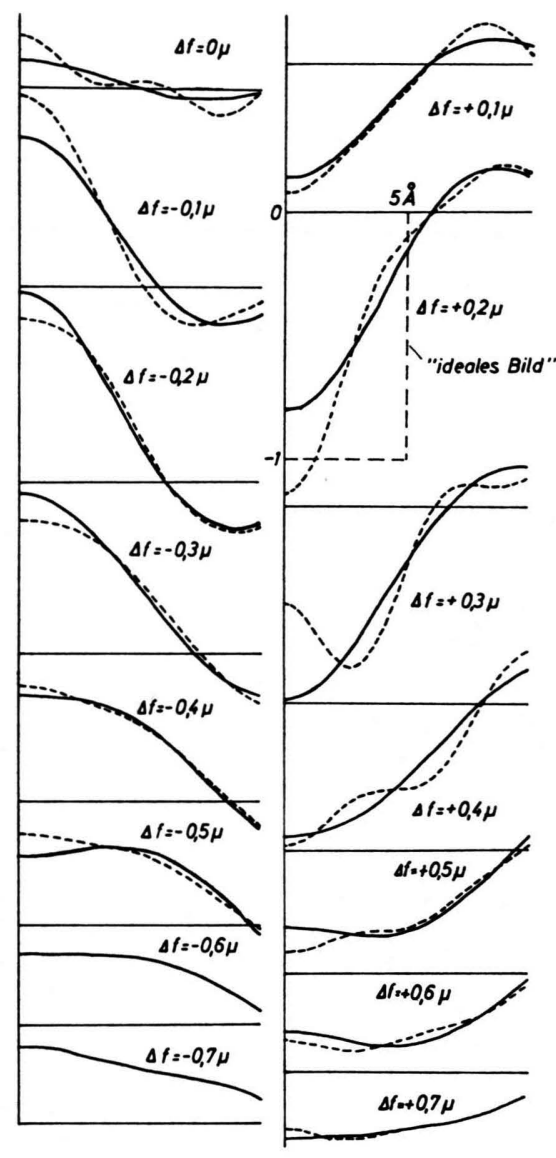

b)

Abb. 5. Die Bildamplitudenfunktion $L\left(r^{\prime}\right)$ in Abhängigkeit von der Bildkoordinate $r^{\prime}$ (horizontal) für a) ein $5 \AA \cdot$, b) $10 \AA \cdot$, c) $20 \AA$ - und d) $40 \AA$-Scheibchen als Phasenobjekt bei verschiedenen Defokussierungen $\Delta f$. Ausgezogene Kurven für eine Apertur von $\alpha=4 \cdot 10^{-3}$, gestrichelte für $8 \cdot 10^{-3}\left(U_{0}=80 \mathrm{kV}\right.$, $C_{\ddot{o}}=4 \mathrm{~mm}$ ). Die gestrichelten Rechtecke in b), c) und d) geben die ,ideale“ Bildamplitude an (Normierung auf 1). In a) muß es heißen $\Delta f=+0,1 \mu$ (statt $f=+0,1 \mu$ ) und in d) $\Delta f=-0,4 \mu$ (statt $f=-0,4 \mu)$.

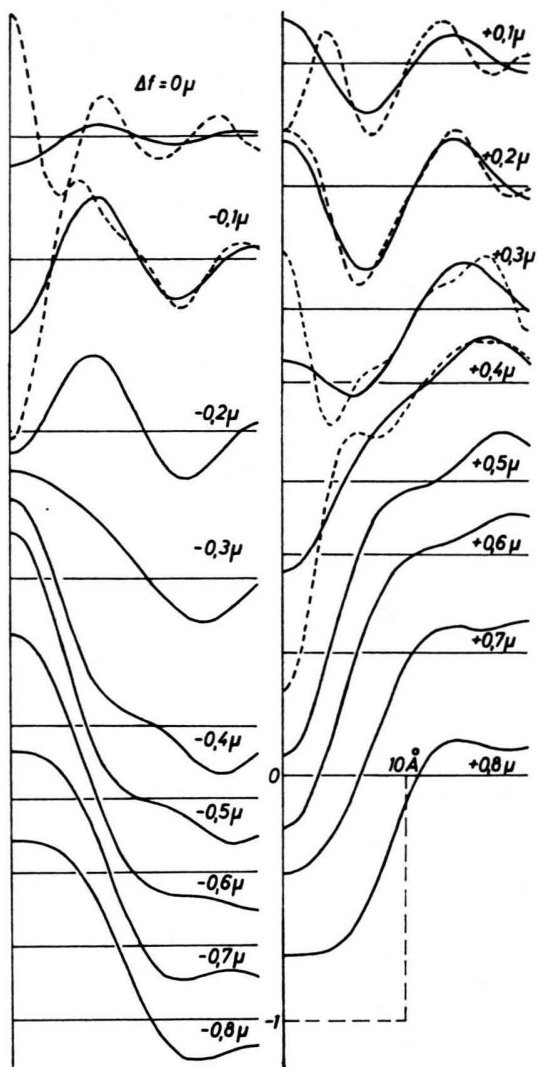

c)

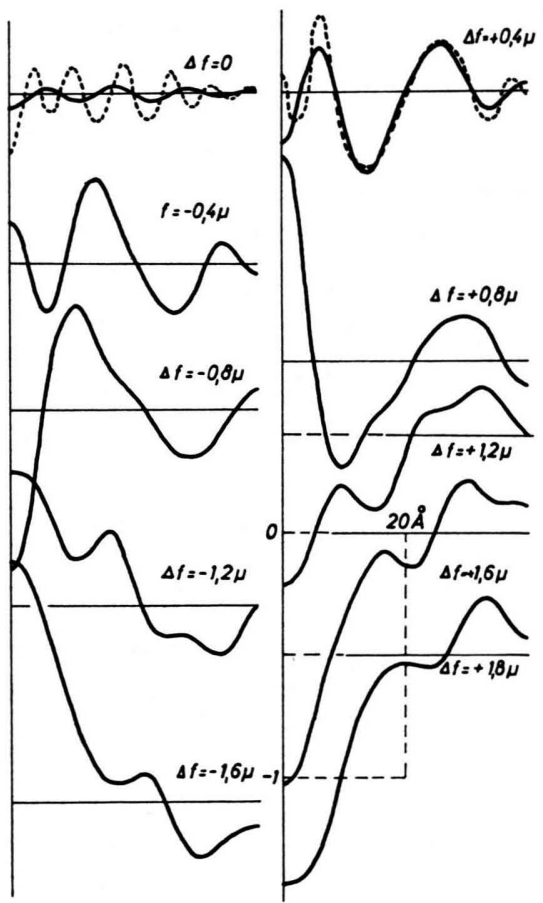

d) 


\section{Quantitative Durchrechnung einer phasenschiebenden Scheibe}

Eine Scheibe als Phasenobjekt mit einer Dicke $D \ll 2 R$ wurde gewählt, weil mit (20) ein einfach auswertbares Integral zur Verfügung steht und auch viele organische Moleküle mit ebener Atomanordnung sich durch eine derartige Scheibe approximieren lassen.

Das Integral in (20) wurde numerisch mit einer elektronischen Rechenmaschine (Zuse Z 23) für verschiedene Werte des Radius $R$, der Defokussierung $\Delta f$ und der Apertur $\alpha$ (spez. $4 \cdot 10^{-3}$, ausgezogene Kurven, und $8 \cdot 10^{-3}$, gestrichelte Kurven in Abb. 5) ausgewertet. Es wurde $C_{\ddot{0}}=4 \mathrm{~mm}$ und $U_{0}=80 \mathrm{kV}$ angesetzt. Ausgewertet wurde der Ausdruck

$$
\begin{aligned}
L\left(r^{\prime}\right) & =\frac{2 \pi R}{\lambda} \\
& \cdot \int_{0}^{\alpha} J_{1}\left(\frac{2 \pi}{\lambda} R \vartheta\right) J_{0}\left(\frac{2 \pi}{\lambda} r^{\prime} \vartheta\right) \sin \Delta(\vartheta) \mathrm{d} \vartheta .
\end{aligned}
$$

Diese Normierung wurde gewählt, weil dann nach (21) bei idealer Phasenschiebung $\left(90^{\circ}\right.$ für alle abgebeugte Strahlung) und ohne Aperturbegrenzung $L\left(r^{\prime}\right)=1$ ist. In Abb. $5 \mathrm{a}-\mathrm{d}$ sind die Rechenergebnisse jeweils gegen $r^{\prime} / R$ aufgetragen. Wenn man sich die Diagramme durch Umklappen um die Ordinate nach links ergänzt denkt, erhält man den Bildamplitudenverlauf oder nach (27) auch den Intensitätsverlauf, falls man das Phasenobjekt radial photometrieren würde. Man ersieht aus den Diagrammen folgende Einzelheiten:

Es genügt nicht, den Phasenkontrast nur für die Bildmitte auszurechnen, da auch die gesamte Intensitätsverteilung im Endbild von Bedeutung ist. Die Diagramme zeigen deutlich die Formänderungen der Intensitätsverteilungen bei wachsender Defokussierung. Das „Bild“ ist mit dem Objekt nicht identisch. Bei stärkeren Defokussierungen resultieren keine „Scheibchenstrukturen“, sondern ein verwaschenes Bild ähnlich eines Arryschen Fehlerscheibchens. (In den Abb. 5 ist gestrichelt das ,ideale Bild“ der Scheibchen eingezeichnet.) Man muß sich jedoch auch die Frage vorlegen, wie das Objektiv ein Objekt bei „idealer Phasenschiebung“ abbilden würde, d. h. falls für alle Streuwinkel $\vartheta$ die Phasenschiebung $\Delta=\pi / 2$ $\left(90^{\circ}\right)$ ist, oder $\sin \Delta=1$ in (20) bzw. (23). Abb. 6 zeigt den so berechneten Einfluß der Aperturbegrenzung auf die Abbildung der Scheibchenstruktur. Sieht

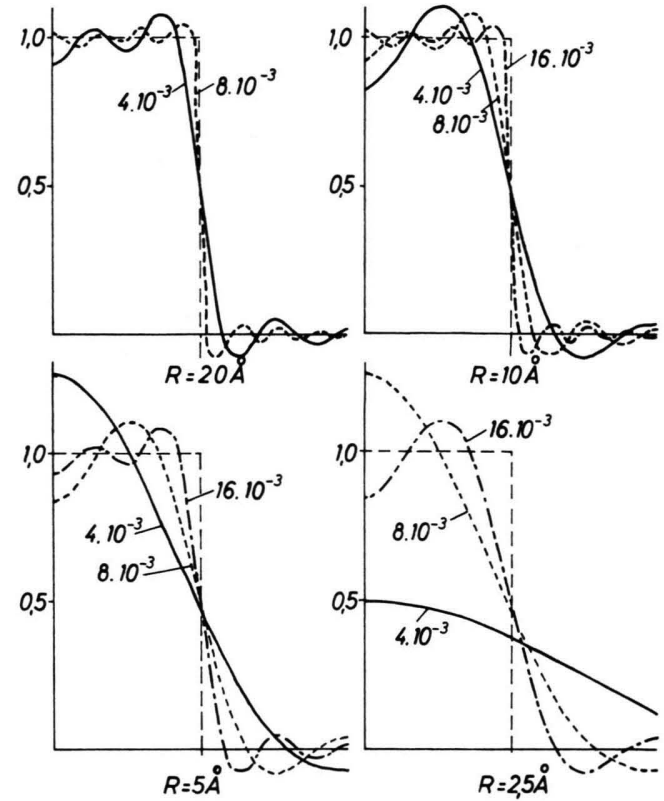

Abb. 6. Bildamplitudenfunktion $L\left(r^{\prime}\right)$ bei idealer Phasenschiebung $\Delta=90^{\circ}$ aller gestreuten Elektronen. Die „Kantenabrundung" erfolgt nur durch den Eingriff der Objektivaperturbegrenzung in der Ebene des 1. Beugungsbildes.

man von den Verhältnissen bei $\operatorname{dem} R=2,5 \AA$ A-Scheibchen ab, welches an der Grenze des Auflösungsvermögens liegt, so werden bei den größeren Scheibchen mit wachsender Apertur befriedigend steile Intensitätsabfälle am Rande erreicht. Während aus der Abb. 6 folgt, daß eine zunehmende Apertur die Güte des Bildes laufend verbessert, gilt dieses nicht bei der Berücksichtigung der realen Phasenschiebung $\Delta$ nach (4). Die schlechte Wiedergabetreue der defokussierten Abbildung beruht also weniger auf einem Einfluß der Aperturbegrenzung. Der Unterschied in den Bildamplituden ist in den Abb. 5 für verschiedene Aperturen sehr gering, deshalb sind nur Werte für $\alpha=4 \cdot 10^{-3}$ und $8 \cdot 10^{-3}$ eingezeichnet. Höhere Aperturen ergaben unter den hier berechneten Bedingungen Kurven, die sich praktisch mit derjenigen für $\alpha=8 \cdot 10^{-3}$ decken. Es sind aber durchaus Fälle denkbar (z. B. geringe Ausdehnung des Phasenobjektes, kleiner Öffnungsfehler und schwache Defokussierung), wo der Phasenkontrast mit wachsender Apertur zunimmt und nicht, wie unten geschildert, konvergiert.

In den Diagrammen, die nur die ausgezogene Kurve für $\alpha=4 \cdot 10^{-3}$ enthalten, liegt kein wesentlicher Apertureinfluß vor. Man kann diese Unempfindlichkeit von der Apertur veranschaulichen, 
wenn man in (23) $\sin \Delta$ durch den komplexen Ausdruck $e^{i \Delta}$ ersetzt und Real- und Imaginärteil mit der EulERschen Beziehung getrennt ausrechnet, indem man von der Apertur 0 beginnend bis $\alpha$ aufintegriert. Jedem Punkt in einem derartigen AmplitudenPhasen-Diagramm (Abb. 7) entspricht eine bestimmte Apertur. Dieses Diagramm zeigt graphisch die Konvergenz von Amplitude und Phasendrehung mit wachsender Apertur. Jeder eingezeichnete Punkt dieses Diagrammes ist ein Schritt bei der numerischen Aufsummation zur Berechnung des Integrales (23). Diese Intervallbreite wurde auch bei der Berechnung der Kurven in Abb. 5 benutzt. Oberhalb $16 \cdot 10^{-3}$ ist die Kurve des Amplituden-Phasen-Diagramms so stark gekrümmt, daß diese in engen spiralförmigen Bahnen um einen Konvergenzpunkt herumläuft. Bei größeren $R$ und größeren Defokussierungen tritt diese Konvergenz schon praktisch bei etwa $4 \cdot 10^{-3}$ auf.

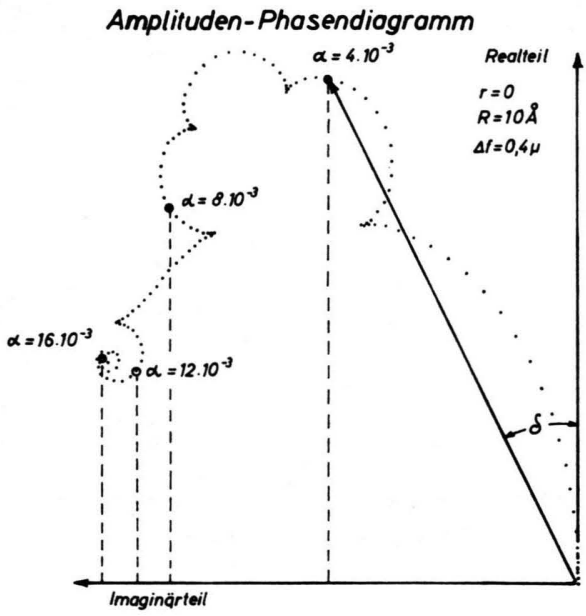

Abb. 7. Darstellung des Verlaufes von $B\left(r^{\prime}\right)$ für ein $20 \AA$ Scheibchen, einer Defokussierung von $\Delta f=0,4 \mu$ und $r^{\prime}=0$ (Bildmitte) bei wachsender Aperturöffnung $\alpha$.

Die Variation der Öffnungsfehlerkonstanten in den Rechnungen zeigte, daß diese bei Teilchendurchmessern größer als $10 \AA$ praktisch keinen Einfluß auf die Rechenergebnisse hat. Dies ist leicht einzusehen, da der Öffnungsfehlerterm in (4) proportional zu $\vartheta^{4}$ geht. Bei den kleinen Streuwinkeln, unter denen die Beugungsmaxima derartig großer Phasenobjekte zu finden sind, ist der Beitrag des Öffnungsfehlers zur Phasenschiebung also sehr gering und der Beitrag proportional $\vartheta^{2}$ der Defokussierung überwiegt bei weitem.

\section{Berücksichtigung des atomaren Aufbaues phasenschiebender Objekte}

Oben wurde der einfachste Fall eines phasenschiebenden Objektes in Form einer Scheibe behandelt. Ebenso elementar ließe sich auch als anderer Modellfall der Phasenkontrast eines kugelförmigen Teilchens berechnen. Scheibchen aus 5-20 $\AA$ Durchmesser sind jedoch bereits aus einer endlichen Zahl von Atomen aufgebaut, so daß der Einfluß dieses Realbaues phasenschiebender Scheibchen zu berücksichtigen ist.

Abb. 8 zeigt z. B., wie Scheibchen von 10 oder $20 \AA$ aus Einzelatomen in dichtester oder quadratischer Packung aufgebaut sind. Im folgenden soll nicht die Bildamplitude $B\left(r^{\prime}\right)$ ausgerechnet werden, sondern die Berechnung beschränkt sich auf $F(\vartheta)$ der Beugungsamplitude dieser Anordnungen. Wie in der kinematischen Theorie der Beugung für Kristallgitter - etwa bei der Berechnung des Strukturfaktors einer Elementarzelle - kann man davon ausgehen, daß sich die Streukurve $F(\vartheta)$ aus dem Produkt der Streufunktion $f(\vartheta)$ des Einzelatoms (10) und der phasenrichtigen Summation über alle $n$ Atome des Scheibchens zusammensetzt:

$$
F(\vartheta)=f(\vartheta) \sum_{k=1}^{n} \exp \left\{2 \pi i\left(\mathfrak{f}-\mathfrak{f}_{0}\right) \mathfrak{r}_{k}\right\} \text {. }
$$

Dieser Ausdruck läßt sich einfacher auswerten, wenn man voraussetzt, daß in bezug auf das Zentralatom mit $\mathfrak{r}_{0}=(0,0)$ die Atome symmetrisch liegen, d. $h$. $\mathrm{zu}$ einem Atom $\mathfrak{r}=(x, y)$ ist auch eines mit $\mathfrak{r}=$ $(-x, y)$ vorhanden. Dann läßt sich $(24)$ zusammenfassen

$$
\begin{aligned}
F(\vartheta) & =f(\vartheta)\left\{1+2 \sum_{k=1}^{l=(n-1) / 2} \cos \left(2 \pi\left(\mathfrak{f}-\mathfrak{f}_{0}\right) \mathfrak{x}_{k}\right)\right\}(25) \\
& =f(\vartheta)\left\{1+2 \sum_{k=1}^{l} \cos \left(\frac{2 \pi}{\lambda}\left(x_{k} \xi+y_{k} \eta\right)\right)\right\}
\end{aligned}
$$

mit $\mathfrak{r}_{k}=\left(x_{k}, y_{k}\right)$ als Position des $k$-ten Atoms und $(\xi, \eta)$ den Winkelkoordinaten in der Beugungsebene $\left(\sqrt{\xi^{2}+\eta^{2}}=\vartheta\right)$.

Abb. 8 zeigt die mit einer elektronischen Rechenmaschine nach (25) berechneten Beugungsdiagramme der eingezeichneten Atomanordnungen in verschiedenen Richtungen. Diese Diagramme sind nichts anderes als Beugungsdiagramme von in ihrer $\mathrm{Ab}$ messung stark begrenzten Flächengittern. Die Hauptmaxima entsprechen den BrAGGschen Reflexionen an den Netzebenen (zu Netzgeraden entartet), welche senkrecht zur betreffenden Richtung liegen. Ihre Posi- 

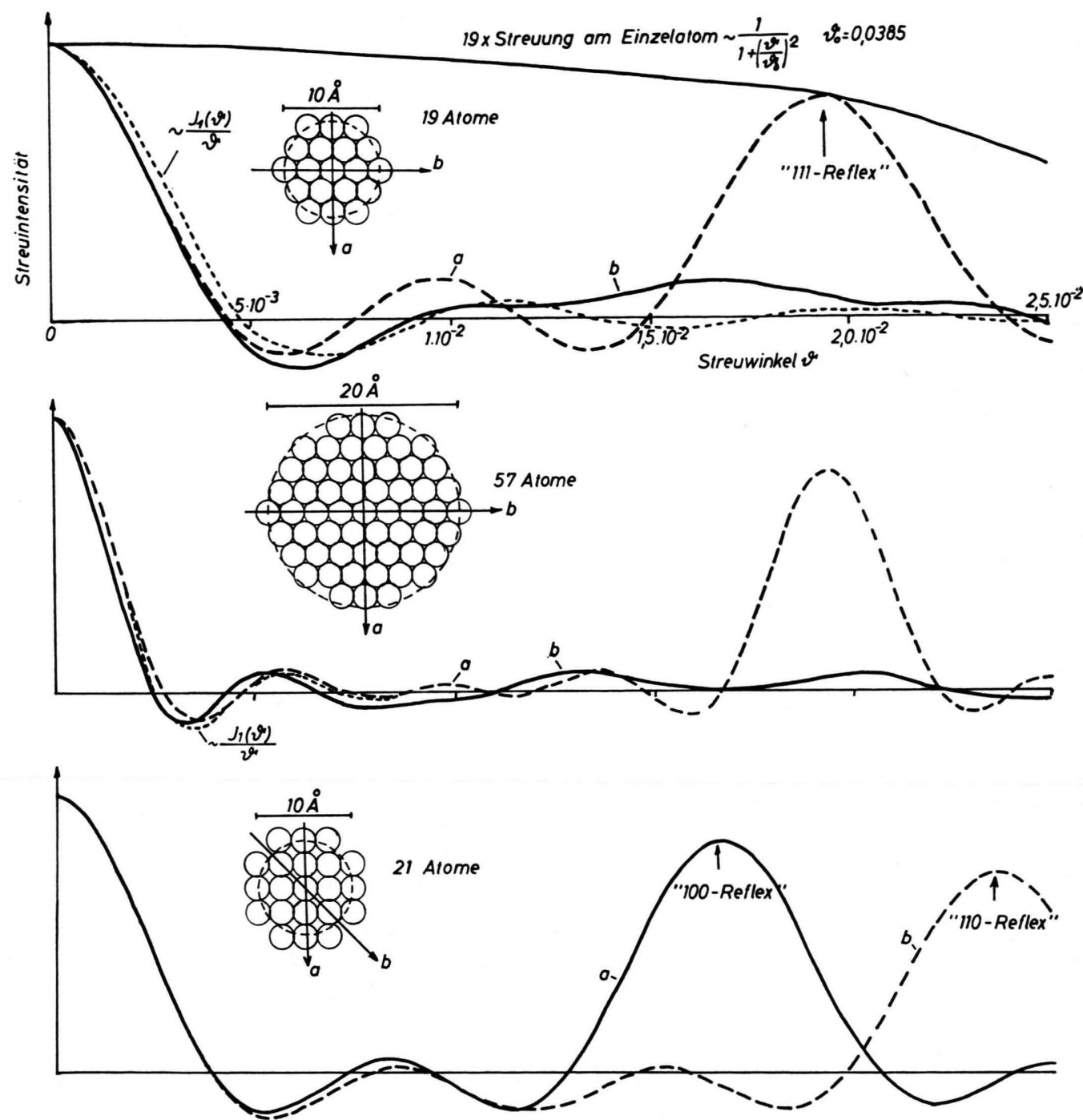

Abb. 8. Die Streukurven $F(\vartheta)$ für phasenschiebende Scheibchen aus einer Monolage von Atomen, in verschiedenen Richtungen a und b zur Atomanordnung. Die fein gestrichelte Kurve resultiert aus der Streuung an einem "homogenen“ Scheibchen.

tion erfüllt die BragGsche Reflexionsbedingung $2 d$ $\sin \Theta=\lambda(\Theta=\vartheta / 2)$. Derartig große Aperturwinkel werden normalerweise jedoch nicht benutzt. Für die Diskussion wichtiger ist der Vergleich mit der Streukurve einer homogenen phasenschiebenden Scheibe mit dem inneren Potential $U_{\mathrm{i}}$ (s. o.) für Aperturen kleiner als etwa $1 \cdot 10^{-2}$. Für diese wurde nach (17) berechnet, daß die Streuamplitude proportional zu $J_{1}(\vartheta) / \vartheta$ ist. Dieser Ausdruck ist zum Vergleich in Abb. 8 eingetragen. Man erkennt, daß die Amplitude und Lage der Maxima, Minima und Nulldurchgänge bei dem $20 \AA$-Scheibchen gut mit der Streukurve des realen Objektes aus Einzelatomen übereinstimmt. Die „kristalline Struktur“ macht sich also erst bei größeren Streuwinkeln im Auftreten von „BRAGGReflexen" bemerkbar. Daß die Übereinstimmung bei der $10 \AA$-Scheibe nicht so genau ist, liegt einfach daran, daß der effektive Radius der Atomanordnung in Wirklichkeit etwas größer als $5 \AA$ ist.

Hiermit ist gezeigt, daß die Annahme eines homogenen phasenschiebenden Objektes mit einem inneren Potential $U_{\mathrm{i}}$ eine gute Näherung ist. Aus (7) kann man ersehen, da $\beta U_{\mathrm{i}}$ und $F(0)$ gemä

$$
F(0)=\frac{2 \pi m e}{\hbar^{2}} \frac{V}{n} U_{\mathrm{i}}
$$

zusammenhängen ( $n=$ Zahl der Atome des Phasenobjektes und $V$ dessen Volumen). 


\section{Aussagen über die Bildintensität bei Phasenkontrast}

Das Integral (23) ist für den Spezialfall eines phasenschiebenden Scheibchens berechnet und in Abb. 5 dargestellt. Es muß nur mit dem Faktor

$$
\frac{\lambda e m U_{\mathrm{i}} D}{2 \pi \hbar^{2}}=\frac{\pi D U_{\mathrm{i}}}{\lambda U_{0}}=\delta
$$

multipliziert werden, um (20) angeben zu können:

$$
B\left(r^{\prime}\right)_{\text {ges }}=A_{0}+A_{0} \delta L\left(r^{\prime}\right) .
$$

Um die gesamte Bildintensität zu erhalten, muß man berücksichtigen, daß bei jedem Phasenobjekt ein Untergrund-Kontrast zu erwarten ist. Das Phasenobjekt liegt $z$. B. entweder auf einer Trägerfolie oder besteht aus einer Inhomogenität (Dichtevariation) in einer homogenen Schicht (s. a. Abb. 1). Definiert man mit $T=I / I_{0}$ die "Transmission" des Objektuntergrundes $\left(I_{0}=\right.$ einfallende Elektronenintensität in $\mathrm{A} / \mathrm{cm}^{2}$ ), so hängt diese von der Objektivapertur $\alpha$ ab (s. u.). Es bedeute $T_{0}$ der Anteil der Transmission, der aus der Streuung in sehr kleine Streuwinkel resultiert (Diskussion s. u.) und $T(\alpha)$ die höhere Transmission, welche sich aus den in Winkel zwischen 0 und $\alpha$ gestreuten Elektronen zusammensetzt, welche die Aperturblende passieren können.

Die gesamte Bildintensität überlagert sich aus diesem Anteil des Objektuntergrundes und dem Phasen- anteil, welchen man durch Quadrieren der Bildamplitude (26) erhält. Da in (26) der zweite Term bedeutend kleiner als $A_{0}$ ist, kann man das Quadrat dieser kleinen Größe vernachlässigen und erhält

$$
\begin{aligned}
\frac{I\left(r^{\prime}\right)}{I_{0}} & =\left(1+2 \delta L\left(r^{\prime}\right)\right) T_{0}+\left(T(\alpha)-T_{0}\right) \\
& =T(\alpha)\left(1+2 \delta L\left(r^{\prime}\right) \frac{T_{0}}{T(\alpha)}\right) .
\end{aligned}
$$

Über $T(\alpha)$ lassen sich quantitative Aussagen mit Hilfe der Lenzschen Streutheorie ${ }^{10}$ gewinnen. Den elastischen Streuanteil erhält man aus (10), wenn man durch Integration von $F^{2}(\vartheta)=\mathrm{d} \sigma_{\mathrm{el}} / \mathrm{d} \Omega$ über den Raumwinkel von $\vartheta=\alpha$ bis $\infty$ alle elastisch gestreuten Elektronen erfaßt, welche von der Aperturblende zurückgehalten werden. Die entsprechende Schwächung der Primärintensität $I_{0}$ in einer Schicht $\mathrm{d} x$ erhält man mit Multiplikation der in dieser Schicht vorhandenen Atome und schließlich die gesamte Transmission durch Integration über die Schichtdicke. Mit dem analogen Ausdruck der Lenzschen Theorie für die unelastische Streuung

$$
\begin{aligned}
\frac{\mathrm{d} \sigma_{\text {unel }}}{\mathrm{d} \Omega} & =\frac{4 Z}{a_{\mathrm{H}^{2} q^{\prime 4}}}\left(1-\frac{1}{\left(1+q^{\prime 2} R^{2}\right)^{2}}\right) \\
\text { mit } \quad q^{\prime 2} & =\frac{4 \pi^{2}}{\lambda^{2}}\left(\vartheta^{2}+\left(\frac{J}{4 e U_{0}}\right)^{2}\right)
\end{aligned}
$$

und $J=$,Ionisationsenergie“ des Atoms ergibt sich die Durchlässigkeit $T=I / I_{0}$ zu

$$
T(\alpha)=\frac{I}{I_{0}}=\exp \left\{-\frac{4 x}{Z x_{\mathrm{a}}}\left[\frac{Z \vdots-1}{4\left(1+\left(\alpha / \vartheta_{0}\right)^{2}\right)}+\ln \left(\sqrt{\left.1+\left(\vartheta_{0} / \alpha\right)^{2}\right)}\right]\right\}=\exp \left\{-x / x_{k}\right\}\right.
$$

Die „Kontrastdicke“ $x_{\mathrm{k}}$ ist diejenige Massendicke, welche den $e$-ten Teil der Intensität (37\%) durch die Aperturblende hindurchläßt. Es bedeuten ferner

$$
x_{\mathrm{a}}=\frac{\pi a_{\mathrm{H}}^{2} A}{N_{\mathrm{L}} Z^{2} R^{2} \lambda^{2}\left(1+\left(e U_{0} / m c^{2}\right)\right)}
$$

die "Aufhellungsdicke" und $\vartheta_{0}$ [s. o. (10)] der „charakteristische Winkel“.

Gl. (30) gilt für den unelastischen Anteil nur für Aperturen größer als $1-2 \cdot 10^{-3}$, weil in (29) unter diesen Bedingungen $\left(\mathrm{J} / 4 \text { e } U_{0}\right)^{2}$ gegenüber $\vartheta^{2}$ zu vernachlässigen ist. Die Aussagen der Lenzschen Theorie lassen sich mit den Experimenten ${ }^{12,13}$ in Einklang bringen, wenn man für ein Element bei einer Strahlspannung $x_{\mathrm{a}}$ und $\vartheta_{0}$ aus dem Experiment durch Ausgleichsrechnung ermittelt. Es genügen dann diese

12 W. Lippert u. W. Friese, V. Intern. Congr. Electron Microscopy Philadelphia, Vol. I, AA-1 [1962].
2 Konstanten, um die Aperturabhängigkeit und den Einfluß der Strahlspannung aus (30) zu berechnen (Ähnlichkeitsrelationen nach LIPPERT ${ }^{12}$ ).

Den Bruchteil der Elektronen $T_{0}$, welcher ungestreut bleibt, erhält man auf folgende Weise. Aus (30) ergibt sich der elastische Gesamtquerschnitt, wenn man $\alpha=0$ setzt. Dies ist für den unelastischen Anteil in (30) nicht erlaubt (s. o.). Es liegen aber von Lenz Berechnungen des Verhältnisses $n$ vom unelastischen zum elastischen Gesamtquerschnitt vor (s. Tabelle), womit

$$
T_{0}=\exp \left\{-\frac{x}{x_{\mathrm{a}}}(1+n)\right\} .
$$

In Abb. 9 ist für einige Beispiele die Abhängigkeit der Transmission von der Apertur dargestellt. Die

13 L. Rermer, Z. Angew. Phys. 13, 432 [1961]. 
Werte auf der Ordinatenachse stellen die $T_{0}$-Werte dar. Es wurden folgende Zahlenwerte benutzt, die für $U_{0}=80 \mathrm{kV}$ aus experimentellen Daten ${ }^{12,13}$ gewonnen wurden:

\begin{tabular}{|c|rc|cc|cc|}
\hline $\begin{array}{c}\text { Ele- } \\
\text { ment }\end{array}$ & $\begin{array}{r}x_{a}\left[\mu \mathrm{g} \cdot \mathrm{cm}^{-2}\right] \\
\text { exp. }\end{array}$ & \multicolumn{2}{|c|}{$\vartheta_{0}$} & \multicolumn{2}{|c|}{$n$} \\
\hline $\mathrm{C}$ & 39 & 24,7 & 0,027 & 0,023 & $3,7(40 \mathrm{kV})$ & 4,24 \\
$\mathrm{Ni}$ & 23 & 14,9 & 0,034 & 0,038 & - & 1,25 \\
$\mathrm{Pt}$ & 17 & 13,1 & 0,034 & 0,054 & - & 0,36 \\
\hline
\end{tabular}

Zum Vergleich sind die aus der Lenzschen Theorie folgenden Werte für $x_{\mathrm{a}}$ und $\vartheta_{0}$ aufgeführt. Der experimentelle Wert für $n$ wurde von uns unter Mitarbeit von FröHLIch mit einem Gegenfeldfilter bestimmt.

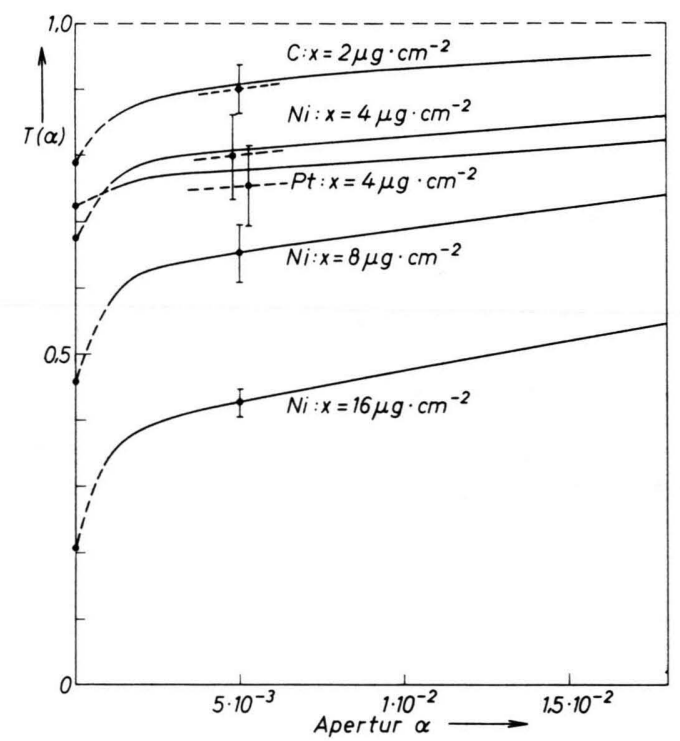

Abb. 9. Die Transmission $T$ als Funktion des Aperturwinkels $a$ für verschiedene Massendicken $x=\varrho D$. Die gestrichelten Kurvenstücke bei $5 \cdot 10^{-3}$ geben den zusätzlichen Streukontrast an, den ein phasenschiebendes Scheibchen von 2,5 $\AA$ Dicke hervorruft. Die eingezeichneten senkrechten Striche zeigen die mögliche Amplitude bei positivem und negativem Phasenkontrast, wenn $L\left(r^{\prime}\right)=1$.

C: $T_{0}=e^{-0,12 x} ; \mathrm{Ni}: T_{0}=e^{-0,098 x} ; \mathrm{Pt}: T_{0}=e^{-0,097 x}$.

Die nach der Theorie von Lenz berechneten Kurven in Abb. 9 gelten jedoch streng nur für gasförmige Objekte, bei denen keine Beeinflussung der Streuung benachbarter Atome durch Interferenzeffekte auftritt. Bei amorphen Objekten weicht jedoch der Kurvenverlauf kaum von demjenigen frei streuender Atome in Abb. 9 ab, weil nach Abb. 2 die Streukurve $F(\vartheta)$ für amorphe Objekte um die Kurve freier Atome oszilliert. Das gleiche gilt für die Durchlässigkeitskurven polykristalliner Aufdampfschichten mit sehr kleinen Kristalliten, deren Abmessungen bedeutend kleiner als die Extinktionsdicke $t_{0}$ der dynamischen Theorie ist, d. h. also der kinematischen Theorie gehorchen. Bei einkristallinen Objekten, die so zum Strahl orientiert sind, daß keine niedrig indizierten BRAGG-Reflexe angeregt sind, ist die Durchlässigkeit höher. Mit der Kontrastdefinition $K=\log \left(I_{\mathbf{0}} / I\right)=-\log T$ ergibt sich experimentell ${ }^{14}$ für $U_{0}=60 \mathrm{kV}$ und $\alpha=4 \cdot 10^{-3}$ unter diesen Beobachtungsbedingungen $K_{\text {krist }} \cong 0,6 K_{\text {amorph }}$ oder $T_{\text {krist }}$ $=\left(T_{\text {amorph }}\right)^{\mathbf{0}, 6}$. Die Elektronen, welche auf diese Weise in kristallinen Objekten durch eine bestimmte Aperturblende mehr hindurchgelassen werden, befinden sich als ungestreute Elektronen im Primärstrahl. In kristallinen Objekten werden außerhalb der BRAGGReflexe überhaupt nur unelastisch gestreute Elektronen und elastisch gestreute Elektronen durch thermisch diffuse Streuung auf Grund der Gitterschwingungen liegen. Diese experimentellen Aussagen sind jedoch zur Zeit die einzigen Informationen über die quantitativen Kontrastverhältnisse in einkristallinen Objekten an Stellen ohne niedrig-indizierte BragGReflexe, an denen etwa Albert u. a. ${ }^{1}$ den Phasenkontrast eingeschlossener Fremdstoffmoleküle beobachteten. Weitere Untersuchungen über die erhöhte Transmission in kristallinen Objekten gegenüber amorphen sind in Vorbereitung. Da die Informationen für einkristalline Objekte demnach bisher noch nicht ausreichen, sind in Abb. 9 und obiger Tabelle die Werte für feinkristalline Ni- bzw. Pt-Schichten eingesetzt.

Die Beleuchtungsapertur $\alpha_{B}$ hat keinen Einfluß auf den Flächenkontrast (30), solange diese bedeutend kleiner als die Objektivapertur $\alpha$ ist. Da in der Praxis die Beleuchtungsapertur je nach Erregung des Kondensors zwischen $10^{-5}$ und $10^{-3}$ liegt und die Objektivapertur zwischen $4 \cdot 10^{-3}$ bis $2 \cdot 10^{-2}$, ist diese Bedingung stets erfüllt. Für den Phasenkontrast hat die Beleuchtungsapertur jedoch einen entscheidenden Einfluß. Die Breite des Primärfleckes in der Beugungsebene (Brennebene des Objektivs) entspricht der Beleuchtungsapertur. Der Phasenkontrast kann sich nur dann ungestört auswirken, wenn die Beleuchtungsapertur kleiner als die durch Beugung verursachte Halbwertsbreite des nullten Hauptmaximums in Abb. 8 ist, also z. B. $\alpha_{\mathrm{B}}<$ ca. $2 \cdot 10^{-3}$ für ein $10 \AA$-Scheibchen. Anderenfalls werden die Beugungsminima- und -maxima verwaschen und sowohl

14 L. Reimer, Lab. Invest. 14, 939 [1965] ; Z. Angew. Phys., in Vorbereitung. 
die Schärfe des Bildes als auch der Phasenkontrast nimmt bei einer Defokussierung ab. Man kann sich dies auch rein geometrisch klar machen. Nach Abb. 5 b liegt das Maximum des Phasenkontrastes bei $\Delta f=0,2 \mu$. Das Objekt muß daher bei dieser Defokussierung um $2 \Delta f \alpha_{\mathrm{B}} \cong 8 \AA$ breiter erscheinen.

In derselben Weise wirkt auch eine Verbreiterung des nullten Hauptmaximums durch unelastische Streuprozesse, die vorwiegend in kleine Winkel erfolgen. Auch die unelastisch gestreuten Elektronen können noch zum Phasenkontrast beitragen. Man kann z. B. beobachten, daß sie zur Intensität der FresneL-Säume beitragen, wenn die Elektronen nach Passieren einer relativ dicken Kohleschicht an einer Kante gebeugt werden. Erst wenn durch Mehrfachstreuung der Primärstrahl einschließlich der unelastisch gestreuten Elektronen divergenter wird, überlagern sich Maxima und Minima der FresneLSäume und es kommt nur zur Ausbildung eines diffusen Beugungssaumes.

Um den Phasenkontrast voll auszunutzen, sollte also die Beleuchtungsapertur $\alpha_{B}$ hinreichend klein sein und die gesamte Dicke des Objektes so gering, daß der Bruchteil $T_{0}$ der ungestreuten Elektronen noch größer als $30 \%$ ist. In Abb. 9 sind die Intensitätsschwankungen eingezeichnet, welche sich nach (27) bei einer Apertur von $5 \cdot 10^{-3}$ und $L\left(r^{\prime}\right)=1$ bei Über- und Unterfokussierung ergeben. Die benutzten $T_{0}$-Werte sind auf der Ordinatenachse zu entnehmen. Für C wurde $U_{\mathrm{i}}=10 \mathrm{~V}$ und für $\mathrm{Ni}$ und $\mathrm{Pt}$ $U_{\mathrm{i}}=20 \mathrm{~V}$ als Näherungswert eingesetzt. Es wird da- bei angenommen, daß ein $2,5 \AA$ dickes Scheibchen auf einer Trägerfolie des betreffenden Materials und der angegebenen Dicke liegt und damit eine zusätzliche Phasenschiebung verursacht. Die gestrichelten Kurvenstücken geben den Kontrast an, den diese zusätzlichen Scheibchen auf Grund der reinen Streuabsorption hervorrufen würden. Bis auf $\mathrm{Pt}$ wären die hierdurch hervorgerufenen Unterschiede in der Transmission so gering, daß sie visuell kaum zu erkennen sind. Durch Phasenkontrast können jedoch so große Unterschiede in der Bildintensität auftreten, daß obige Phasenobjekte klar zu erkennen sind. Aus den Abb. 5 ist jedoch zu ersehen, daß für Phasenobjekte mit Durchmessern kleiner als $10 \AA L\left(r^{\prime}\right)$ weit unter 1 bleibt.

Das Ziel der vorliegenden Arbeit ist es, den $\mathrm{Zu}$ sammenhang zwischen dem Phasenkontrast durch Defokussierung und der Elektronenstreutheorie aufzuzeigen. Es werden von uns Versuche unternommen, die Theorie mit Experimenten zu vergleichen, über die später berichtet werden soll. Die Schwierigkeiten liegen in der Wahl geeigneter Objekte, welche nicht zu klein sein sollen, da sonst die Photometrierung der photographischen Platte erschwert wird. Wir haben deshalb die Versuche zunächst mit FerritinMolekülen begonnen, die einen Durchmesser von $50 \AA$ besitzen und wegen des Gehaltes an Fe-Atomen auch im Fokus noch klar zu erkennen sind.

Mein besonderer Dank gilt Herrn Dr. L. Albert für ausführliche Diskussionen dieser Arbeit.

\section{BERICHTIGUNGEN}

Zu Tн. Heumann und D. Primas, Zum Isotopieeffekt der Diffusion von Wasserstoff und Deuterium in Reinsteisen, Z. Naturforschg. 21 a, 260 [1966].

Auf Seite 264, rechte Spalte, Tab. 1, müssen die Zahlen der dritten Spalte 24, 17, 890 , 631 und die der letzten Spalte 0,268, 0,327, 0,075, 0,077 lauten. Die Worte „recht gut“ in der vorletzten Zeile unter Tab. 1 sind durch „mäßig“ zu ersetzen.

Zu B. Schrader und W. Meier, Laser-Mikro-Anordnungen für die Raman-Spektroskopie von Flüssigkeiten und Kristallpulvern, Z. Naturforschg. 21 a, 480 [1966].

Auf S. $481 \mathrm{muß}$ es in der Unterschrift zu Abb. 4 (linke Spalte) und im Text (rechte Spalte, erster Absatz) heißen: Triphenylcyclopropenyliumbromid. 